\title{
On coupling methods used to simulate the dynamic characteristics of heavy ground vehicles subjected to crosswind
}

\author{
Tural Tunay ${ }^{\mathrm{a}, \mathrm{b}, \mathrm{c}, *}$, Ciarán J. O'Reilly ${ }^{\mathrm{a}, \mathrm{b}}$, Lars Drugge ${ }^{\mathrm{a}, \mathrm{b}}$ \\ ${ }^{a}$ KTH Royal Institute of Technology, Department of Aeronautical and Vehicle \\ Engineering, Teknikringen 8, 10044 Stockholm, Sweden \\ ${ }^{b}$ The Centre for ECO ${ }^{2}$ Vehicle Design at KTH, Teknikringen 8, 10044 Stockholm, \\ Sweden \\ ${ }^{c}$ Adana Alparslan Turkes Science and Technology University, Faculty of Engineering, \\ Department of Mechanical Engineering, 01180 Adana, Turkey
}

\begin{abstract}
Ground vehicles often encounter turbulent flows with wide range of scales, e.g. crosswind gusts, when moving on-road conditions. Crosswinds can be considered as an important factor in heavy vehicle's roll-over accident due to their relatively high center of gravity and lateral surface area. However, there are few studies which investigate effects of crosswinds on heavy vehicles. Furthermore, existing studies have generally focused on lateral or yaw dynamics of heavy vehicles and neglected their roll dynamics. Therefore, effects of crosswinds on heavy ground vehicles should be understood in all aspects in order to design less wind-sensitive vehicles especially at early stages of their design processes. Inherently, crosswind studies require a multidisciplinary approach which involves coupling between aerodynamics and vehicle dynamics. In the present study, investigation of effects of the coupling
\end{abstract}

\footnotetext{
${ }^{*}$ Corresponding author

Email address: ttunay@atu.edu.tr (Tural Tunay)
} 
methods, e.g. one-way and two-way couplings, between aerodynamics and vehicle dynamics simulations are aimed by considering both lateral and roll dynamics of heavy vehicles which are subjected to crosswind gust. Vehicle dynamics and aerodynamics results are presented. Results of the one-way coupled simulations, in general, overestimate the absolute maximum values of the vehicle dynamics results. However, differences in lateral displacement results $\Delta y_{\max }$ are less than $0.2 \mathrm{~m}$ for the studied case.

Keywords: Coupled simulations, Crosswind, GTS, Heavy ground vehicles, IDDES

\section{Introduction}

2

In previous methods of classical aerodynamics, design procedures of ground vehicles have generally been limited to optimization of their external geometries at either zero yaw angle or certain steady yaw angles. Therefore, in general, effects of the unsteady aerodynamic forces on the vehicles are ignored. Hence, over the last century, as a result of the investigations on the vehicle aerodynamics, drag force has been decreased by $70 \%$, but the yaw moment at crosswinds of 20 degrees has been increased by $150 \%$ (Kee et al., 2014). In fact, depending on dynamic properties of the vehicle and behaviour of the driver, transient aerodynamic excitations, such as caused by crosswind gusts, may be enhanced or damped (Wojciak, 2012). Therefore, a reliable prediction of the crosswind stability of the vehicles early in their development processes is essential in order to avoid costly modifications after the production or even recalls (Theissen, 2012).

Nowadays, understanding of the crosswind stability is also essential for 
Table 1: Previous studies that conduct two-way coupling between aerodynamics and vehicle dynamics equations

\begin{tabular}{|c|c|c|}
\hline Authors & Title & Vehicle Type \\
\hline $\begin{array}{l}\text { Nakashima et } \\
\text { al. }(2013)\end{array}$ & $\begin{array}{l}\text { Coupled analysis of unsteady aerodynamics and vehi- } \\
\text { cle motion of a road vehicle in windy conditions }\end{array}$ & Heavy vehicle \\
\hline $\begin{array}{l}\text { Ishioka et al. } \\
(2015)\end{array}$ & $\begin{array}{l}\text { Coupled } 6 \text { Dof Motion and Aerodynamics Simulation } \\
\text { of Road Vehicles in Crosswind Gusts }\end{array}$ & Passenger car \\
\hline $\begin{array}{l}\text { Winkler et al. } \\
(2016)\end{array}$ & $\begin{array}{l}\text { Coupling aerodynamics to vehicle dynamics in tran- } \\
\text { sient crosswinds including a driver model }\end{array}$ & Heavy vehicle \\
\hline $\begin{array}{l}\text { Carbonne et } \\
\text { al. }(2016)\end{array}$ & $\begin{array}{l}\text { Use of Full Coupling of Aerodynamics and Vehicle } \\
\text { Dynamics for Numerical Simulation of the Crosswind } \\
\text { Stability Ground Vehicles }\end{array}$ & $\begin{array}{l}\text { Passenger car } \\
\text { Heavy vehicle }\end{array}$ \\
\hline $\begin{array}{l}\text { Forbes et al. } \\
(2016)\end{array}$ & $\begin{array}{l}\text { A Fully Coupled, } 6 \text { Degree-of-Freedom, Aerodynamic } \\
\text { and Vehicle Handling Crosswind Simulation using the } \\
\text { DrivAer Model }\end{array}$ & Passenger car \\
\hline $\begin{array}{l}\text { Nakasato et } \\
\text { al. (2017) }\end{array}$ & $\begin{array}{l}\text { Coupled } 6 \text { DoF Motion and Aerodynamic Crosswind } \\
\text { Simulation Incorporating Driver Model }\end{array}$ & Passenger car \\
\hline $\begin{array}{l}\text { Huang et al. } \\
(2017)\end{array}$ & $\begin{array}{l}\text { Transient Aerodynamics simulations of a road vehicle } \\
\text { in the crosswind condition coupled with the vehicle's } \\
\text { motion }\end{array}$ & Passenger car \\
\hline $\begin{array}{l}\text { Huang et al. } \\
(2017)\end{array}$ & $\begin{array}{l}\text { Coupled Analysis of Unsteady Aerodynamics and Ve- } \\
\text { hicle Motion of a Passenger Car in crosswind Condi- } \\
\text { tion }\end{array}$ & Passenger car \\
\hline $\begin{array}{l}\mathrm{Li} \text { et al. } \\
(2018)\end{array}$ & $\begin{array}{l}\text { Coupled analysis of vehicle stability in crosswind on } \\
\text { low adhesion road }\end{array}$ & Passenger car \\
\hline $\begin{array}{l}\text { Huang et al. } \\
(2019)\end{array}$ & $\begin{array}{l}\text { Investigation of vehicle stability under crosswind con- } \\
\text { ditions based on coupling methods }\end{array}$ & Passenger car \\
\hline
\end{tabular}
architecture give rise to new combinations of basic parameters compared to standard vehicle layouts, e.g. mass distribution and vehicle dimensions, which may have a negative impact on the crosswind stability (Favre, 2011). Besides, there are recent studies on developing new generation vehicles with motor-driven power steering systems in which crosswind events are detected to assist steering torque in an automated manner (Kim et al., 2014). ditions based on coupling methods

the next generation electric vehicles or hybrid drive vehicles since their new

Inherently, investigations of the dynamics of ground vehicles which are 
subjected to crosswind gusts include a multidisciplinary approach in which there is a coupling between aerodynamics and vehicle dynamics simulations. In the previous literature, the number of investigations employing fully coupled, i.e. two-way coupled, simulation of aerodynamics and vehicle dynamics are very limited as presented in Table 1. In general, their main focuses are on lateral or yaw dynamics of passenger cars while investigating effects of different coupling methods or differences between results of quasi-steady and unsteady approaches. It is known that heavy vehicles are subjected to fatal roll-over accident due to their high centre of gravity and lateral surface area. Therefore crosswinds can be considered as an important factor in fatal rollover accidents of heavy vehicles (Cheli et al., 2011a,b). Besides, Tunay et al. (2019) have shown that there are significant differences between results of the two-way coupled simulations considering no-roll and roll motions which are conducted on a heavy ground vehicle. However, despite the importance of consideration of roll dynamics, none of the previous studies has investigated effects of the crosswind on heavy ground vehicles by considering the roll dynamics of the vehicles with different coupling methods together. In the previous literature, Nakashima et al. (2013) have initially conducted a two-way coupled analysis of the simplified truck geometry by ignoring the roll dynamics. In their conclusion, certain differences have been identified in the vehicle path and the yaw angle between the quasi-steady and the fully coupled analyses. After that, Ishioka et al. (2015) have revealed differences between the results of one-way and two-way coupling approaches. Winkler et al. (2016) have conducted a more detailed study and they have stated that one-way coupling approach overestimate the aerodynamic loads 
and accordingly lateral motion of the vehicle, i.e. one-way coupling with no driver predicts $7.3 \%$ larger deviation from the original path. They have concluded that two-way coupling is needed when large yaw angles are expected. Furthermore, Carbonne et al. (2016) have explained the discrepancies between one-way and two-way coupling approaches with progressive change in yaw angle. Forbes et al. (2016) have presented that although there are considerable difference in lateral displacement of $9 \%$ and yaw angle of $11 \%$ between unsteady and quasi-steady approaches, variations in these parameters are minimal in between the results of one-way and two-way coupled unsteady approaches. Therefore, they decided that one-way coupling is sufficient. Although the conclusion of Forbes et al. (2016) seems to conflict with the conclusion of Winkler et al. (2016), it should be noted that classes of the vehicle model which are employed in their studies are different. Whereas Winkler et al. (2016) have investigated flow around a heavy vehicle model, i.e. GTS model, Forbes et al. (2016) have investigated flow around a passenger car model, i.e. DrivAer model. Carbonne et al. (2016) have compared corresponding results between GTS heavy vehicle model and Windsor passenger car model. They have observed that one-way coupling over predicts the total deviation by only $1 \%$ for the Windsor vehicle model. They have stated that the location of centre of gravity of the Windsor model employed in their study is close to the centre of pressure which leads to low values of yaw moment. Therefore, it can be concluded that two-way coupling is needed in cases where high yaw angles are experienced.

In order to design less wind sensitive vehicles early in the development process, industrial applications need a sufficient fidelity and low computa- 
tionally demanding numerical methods. Therefore, the main motivation in the present study is devoted to investigate effects of different coupling methods on the solutions, e.g. one-way and two-way coupling between aerodynamics and vehicle dynamics equations. Accordingly, because understanding of dynamic characteristics of heavy ground vehicles which are subjected to crosswind gusts is essential due to their tendency to roll-over accidents, a simplified heavy vehicle model, GTS, is employed. Details of the coupling methods and other numerical models employed in the study are given in the Methods section. Obtained vehicle dynamics and aerodynamics results are presented and discussed in detail in the Results and Discussion section.

\section{Methods}

\subsection{Coupling Methods}

For the purpose of comparison between the coupling methods, in the present study, both one-way and two-way couplings between aerodynamics and vehicle dynamics simulations are performed. Therefore, three different coupling methods are employed, namely two-way coupling, one-way coupling with moving vehicle and one way coupling with fixed vehicle.

In every time step of the two-way coupling method, first, aerodynamics equations and then, vehicle dynamics equations are solved. Crosswind is introduced in the solution of the aerodynamics equations with the aid of boundary conditions. Coupling between the simulations are performed by exchanging force and moments results of aerodynamics simulation to vehicle dynamics simulation and velocity results of the vehicle dynamics simulation to aerodynamics simulation. The computation proceeds to next time step 
after performing five inner iterations in each time step of the simulation. Total number of the time steps used in the simulation is 3667. Steps followed in each iteration of the two-way coupling method are illustrated in Fig. 1. Additionally, representation of the computational flow domain in the two-way coupling method is given in Fig. 2.

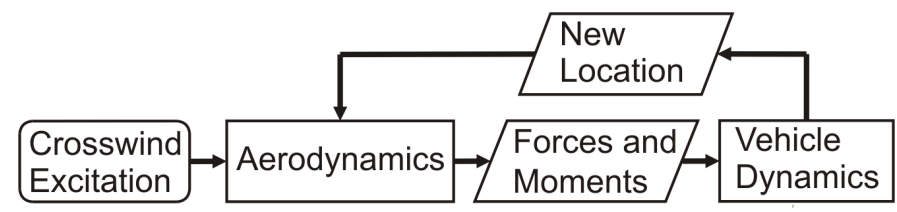

Figure 1: Schematic representation of the two-way coupling method.

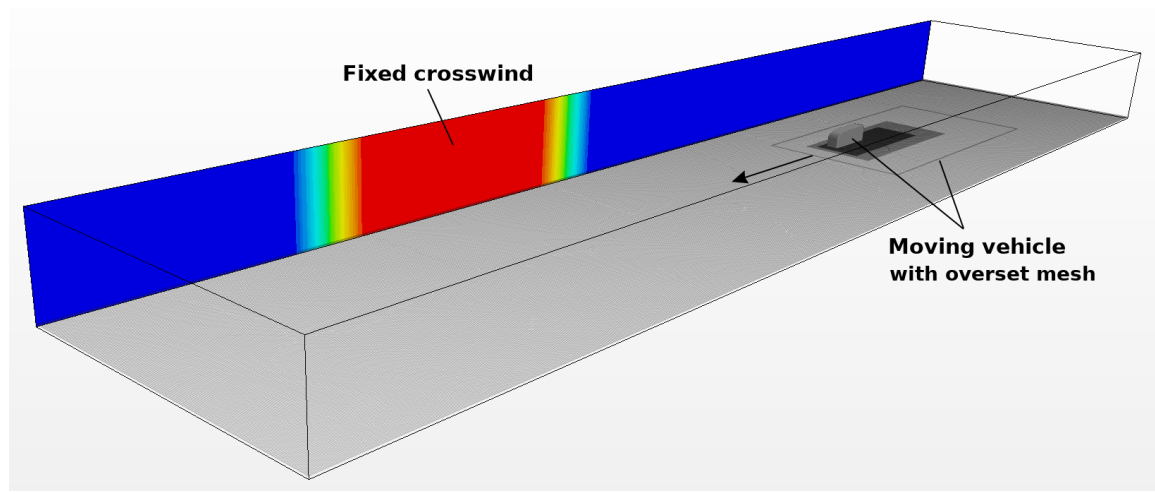

Figure 2: Representation of the computational flow domain in two-way coupling method.

On the other hand, in the one-way coupling with fixed vehicle simulation, closed loop feedback on updated location of the vehicle from the vehicle dynamics equations to aerodynamics equations are no longer used. Accordingly, because there is no update between vehicle dynamics and aerodynamics simulations during the computation, overset mesh is not employed in aerodynamics simulation of the one-way coupled with fixed vehicle simulation. 


\begin{tabular}{|c|c|c|}
\hline Exci & & $\rightarrow \begin{array}{l}\text { Forces and } \\
\text { Moments }\end{array} \rightarrow \begin{array}{l}\text { Vehi } \\
\text { Dyna }\end{array}$ \\
\hline
\end{tabular}

Figure 3: Schematic representation of the one-way coupling method.

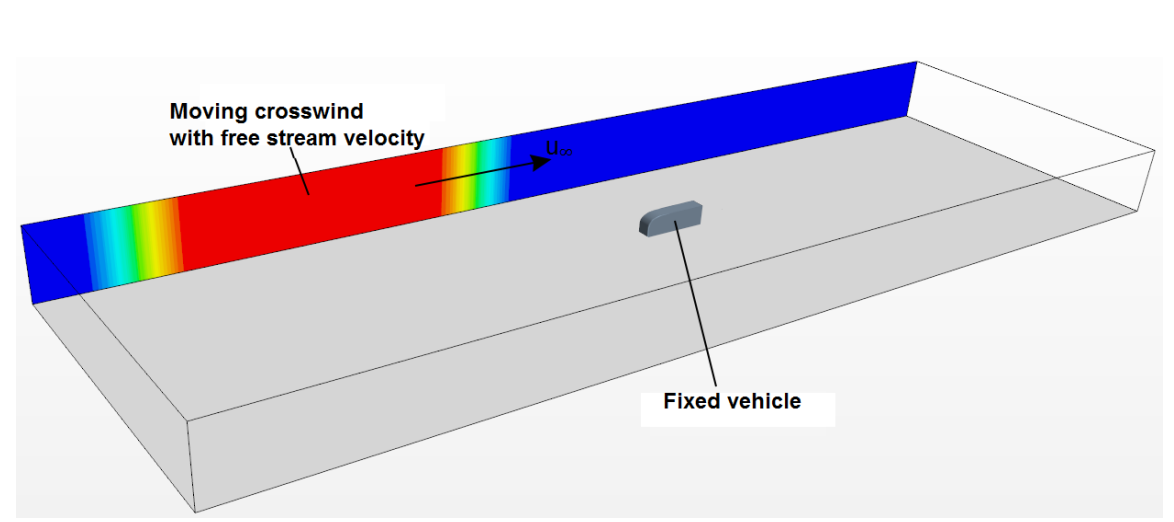

Figure 4: Representation of the computational flow domain in conventional one-way coupling method.

Steps followed in each iteration of the one-way coupled with fixed vehicle simulation and corresponding aerodynamic computational flow domain are presented in Figs. 3 and 4 respectively.

Besides employing two different ways of conventional coupling methods which are explained above, to asses effects of using overset mesh in improving results obtained in one-way coupling method, an additional modified version of the one-way coupling method in which moving vehicle in the flow domain with the aid of overset mesh is employed. Therefore, in the third method, oneway coupling with the same computational flow domain which is employed in the two-way coupling method is used. 


\subsection{Vehicle Model}

In the present study, the Ground Transportation System (GTS) which is designed by Sandia National Laboratory (Gutierrez et al., 1996) is employed as a vehicle model. The GTS is a $1 / 8$ scaled simplified heavy vehicle model. The original GTS is shortened to resemble a bus geometry as presented in Fig. 5. Additionally, wheels under the GTS have been excluded in CFD simulations. On the other hand, in the vehicle dynamics simulations, the full scale GTS vehicle model is employed.

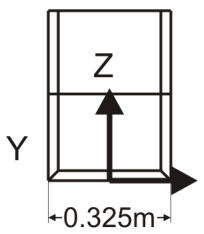

Front view

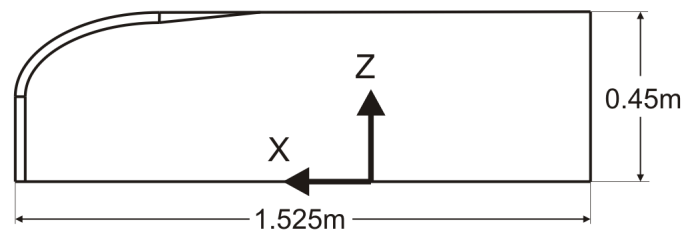

Side view

Figure 5: GTS Vehicle model

\subsection{Crosswind Profile}

Crosswinds are unsteady on-road flow conditions which have wide range of scales and they stem from unsteady flow due to turbulence in natural wind, vehicle velocity changes itself and unsteady wakes of other vehicles and traversing through a steady spatial wind distribution produced by road side-obstacles (Sims-Williams, 2011). Their spectral energy drops at a few $\mathrm{Hz}$ and relevant wind gusts have $10 \mathrm{~m}$ to $80 \mathrm{~m}$ wavelengths that cause a sinusoidal variation in yaw angle (Wojciak, 2012; Theissen, 2012).

In the previous literature, various approaches have been used to simulate effects of crosswinds on ground vehicles. These approaches are grouped in 


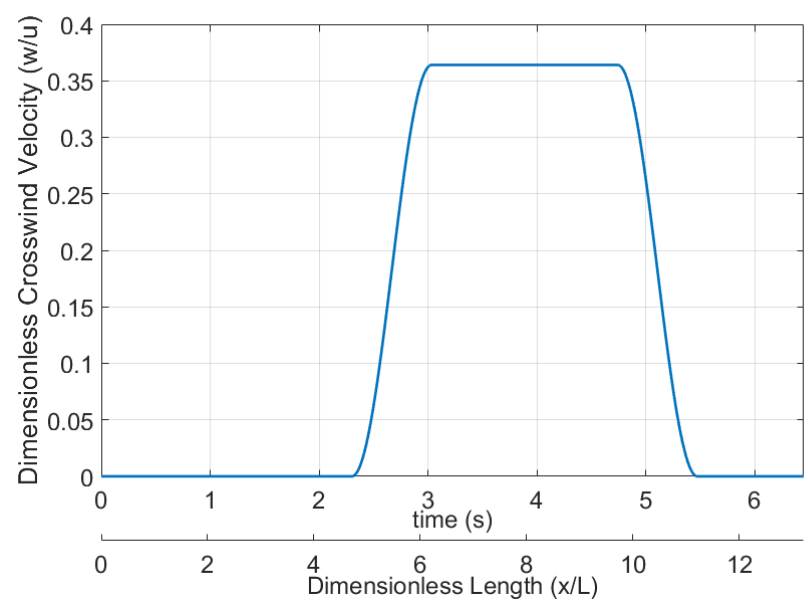

Figure 6: Crosswind profile

the study of Sims-Williams (2011) as steady crosswinds (Guilmineau et al., 2013), dynamic rotation of the vehicle model (Mansor and Passmore, 2008), isolated extreme crosswinds (Volpe et al., 2014), stationary turbulence-small scales (Watkins, 1990) and stationary turbulence-central scales (Schrock et al., 2009). All of the methods listed above have some advantages and disadvantages. Watkins et al. (1992) show that dynamic model rotation cannot allow the correct reproduction of the conditions experienced by a vehicle traversing a generic cross wind gust. Nevertheless, this technique can be instructive in assessing the range of scales of importance. On the other hand, Sims-Williams (2011) states that isolated, extreme transient crosswinds, coupled with the vehicle aerodynamic response, handling and driver reaction, pose a safely risk and it is generally suitable only late in the development cycle.

In the present study, the isolated extreme crosswind model (Sims-Williams, 
2011) which has a trapezoidal profile with cosine inlet and exit profile is used to simulate the on-road crosswind gust. Its length is 5 times the length of the GTS. Maximum magnitude of the crosswind velocity is selected as $9 \mathrm{~m} / \mathrm{s}$ so as to generate maximum 20 degree yaw angle of the incoming velocity which is $25 \mathrm{~m} / \mathrm{s}$. The other details of the crosswind model are given in Fig. 6. This crosswind profile is also employed in many other studies in which they have used two-way coupled simulations, e.g. in the study of (Winkler et al., 2016), (Carbonne et al., 2016), (Forbes et al., 2016), (Nakasato et al., 2017) and (Li et al., 2018).

The crosswind profile used in the present study is exactly similar to the one used in the study of (Winkler et al., 2016). In the two-way coupled simulation and one-way coupled simulation with moving vehicle simulation, the velocity profile of the crosswind is defined in Eq. 1 . Here, $w_{\max }$ is the maximum lateral velocity magnitude of the crosswind, $\Delta x_{\text {slope }}$ is the length of the transient inlet and exit part of the crosswind which is 1.5 times the length of the GTS, $x_{0}$ is the distance between the starting location of the crosswind and the origin of the coordinate system and $\Delta x_{c w}$ is the length of the crosswind which is five times the length of the GTS. 


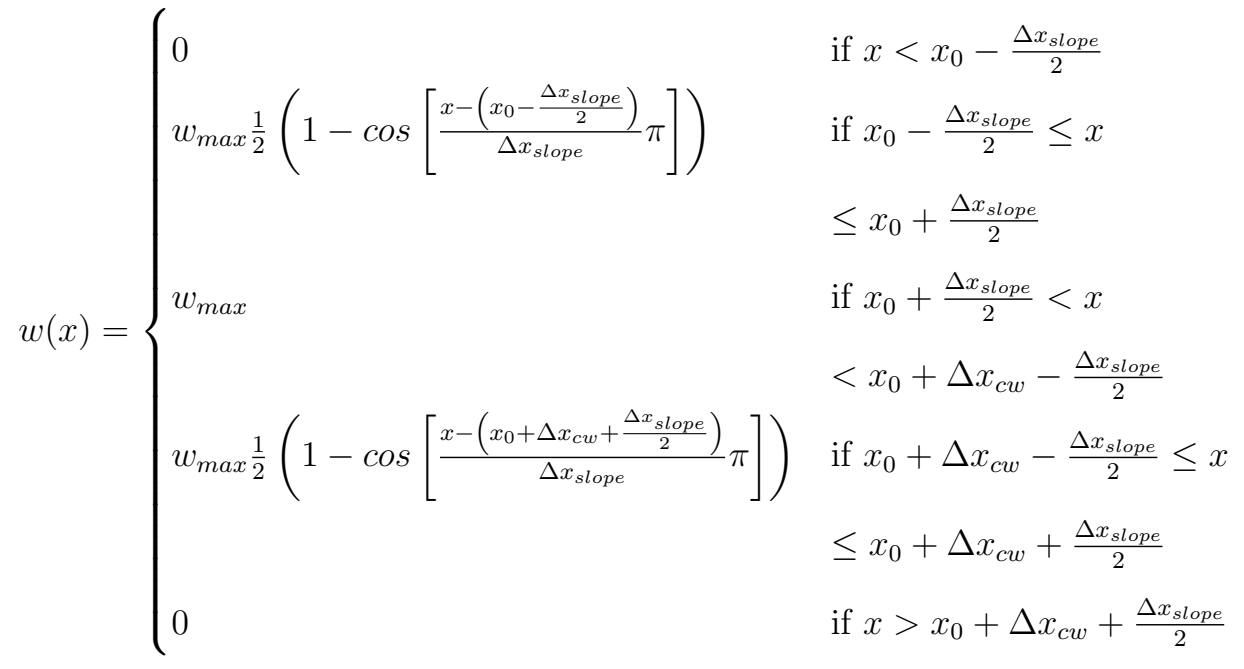

169 Similarly, in the one-way coupled simulation with fixed vehicle, the ve170 locity profile of the crosswind is defined in Eq. 2 . Here, $w_{\max }$ and $x_{0}$ are the same as the definitions given in the two-way coupled simulation, ST is the period of the cosine function to define transient inlet and exit part of the crosswind which is $\left(1.5 L_{G T S}\right) / u_{\infty}$ length of the GTS, $\Delta t_{c w}$ is the time of the 174 crosswind which correspond to $\left(5 L_{G T S}\right) / u_{\infty}$ and $t_{0}$ is the initial time. 


$$
w(x, t)= \begin{cases}0 & \text { if } t<\left(\frac{x-x_{0}}{u_{\infty}}+t_{0}-\frac{S T}{2}\right) \\ w_{\max } \frac{1}{2}\left(1-\cos \left[\frac{\left(x-x_{0}\right)-u_{\infty}\left[t-\left(t_{0}-\frac{S T}{2}\right)\right]}{u_{\infty} S T} \pi\right)\right. & \text { if }\left(\frac{x-x_{0}}{u_{\infty}}+t_{0}-\frac{S T}{2}\right) \leq t \\ w_{\max } & \leq\left(\frac{x-x_{0}}{u_{\infty}}+t_{0}+\frac{S T}{2}\right) \\ & \text { if }\left(\frac{x-x_{0}}{u_{\infty}}+t_{0}+\frac{S T}{2}\right)<t \\ & <\left(\frac{x-x_{0}}{u_{\infty}}+t_{0}+\Delta t_{c w}-\frac{S T}{2}\right) \\ w_{\max } \frac{1}{2}\left(1-\cos \left[\frac{\left(x-x_{0}\right)-u_{\infty}\left[t-\left(t_{0}+\Delta t_{c w}+\frac{S T}{2}\right)\right]}{u_{\infty} S T} \pi\right]\right) & \text { if }\left(\frac{x-x_{0}}{u_{\infty}}+t_{0}+\Delta t_{c w}-\frac{S T}{2}\right) \leq t \\ & \leq\left(\frac{x-x_{0}}{u_{\infty}}+t_{0}+\Delta t_{c w}+\frac{S T}{2}\right) \\ 0 & \text { if } t>\left(\frac{x-x_{0}}{u_{\infty}}+t_{0}+\Delta t_{c w}+\frac{S T}{2}\right)\end{cases}
$$

\subsection{Aerodynamics}

Incompressible continuity and Navier-Stokes equations are used to solve the flow field around the GTS vehicle model. The flow is simulated by using SST k- $\omega$ Detached Eddy Simulation model (DES) with the Improved Delayed Detached Eddy Simulation (IDDES) formulation. IDDES combines Delayed Detached Eddy Simulation model (DDES) with an improved RANSLES hybrid model aimed at wall modelling in LES depending on the grid resolution (Shur et al., 2008).

Generation of the meshes and solution of the governing equations are done by using the commercial CFD software StarCCM+ version 13.02. In the computations, finite volume method and segregated solver are used. HybridBounded Central Differencing (BCD) scheme is used for the convective discretization of the governing equations in which BCD is used in the separated areas and a high-order upwind scheme in the flow-attached and free stream areas. A second order implicit unsteady scheme is used as a time discretization. The resultant scalar system of equations are solved by using Algebraic 
Multigrid (AMG) linear solver.
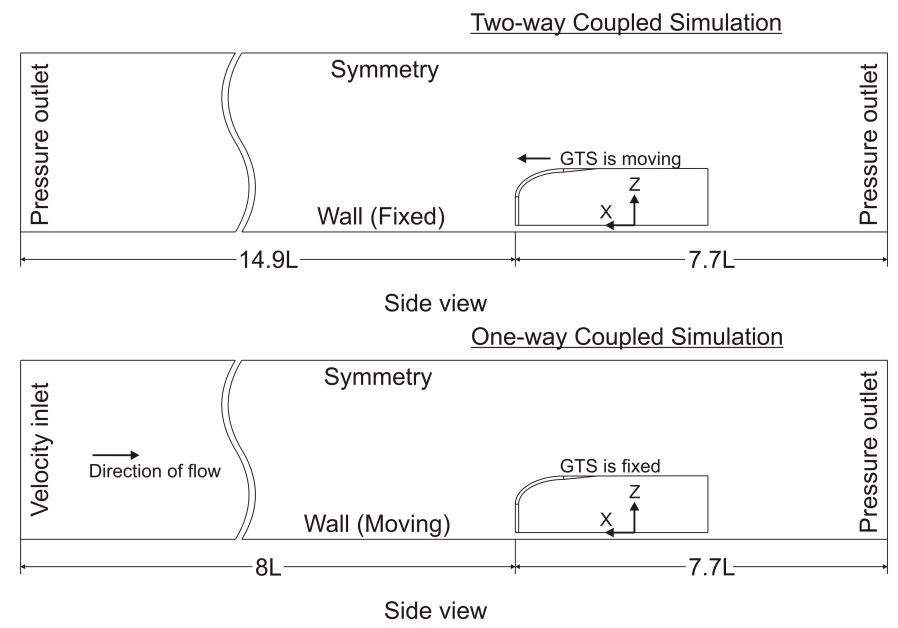

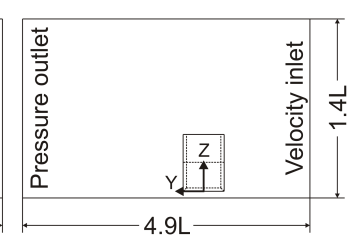

Rear view

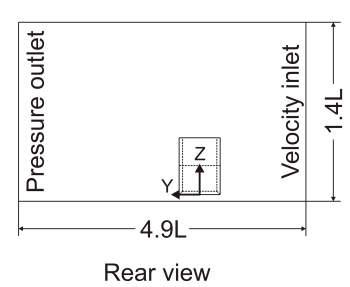

Figure 7: Boundary conditions and dimensions of the computational flow domains.

Boundary conditions and dimensions of the computational domains applied in both two-way and one-way coupled simulations are presented in Fig. 7. Crosswind is introduced to the flow domains with the aid of velocity inlet boundary condition on the right lateral surface of the flow domains. Details of the crosswind profile are given in the previous section. In the two-way coupled simulation, surfaces named as inlet, outlet and left lateral surfaces are set to pressure outlet boundary condition, which assumes atmospheric pressure on the boundary surface. On the other hand, inlet surface of the one-way coupled with fixed vehicle simulation is set to velocity inlet boundary condition, in which a uniform velocity with $91.6 \mathrm{~m} / \mathrm{s}$ is assumed. Ground of the flow domain and surfaces of the GTS are treated as no-slip boundary condition in all simulations. Additionally, whereas ground is set to move with free stream flow velocity in one-way coupled simulation with fixed vehicle, the GTS is set to move with $91.6 \mathrm{~m} / \mathrm{s}$ in the two-way coupled simulation and 
one-way coupled simulation with moving vehicle. The velocity of $91.6 \mathrm{~m} / \mathrm{s}$ corresponds to $R e_{w}=2 \times 10^{6}$ in accordance to the computational study of Unaune et al. (2005) and wind tunnel measurements of Croll et al. (1996) and Storms et al. (2001). Upper surfaces of the flow domain in all simulations are set to symmetry boundary condition.

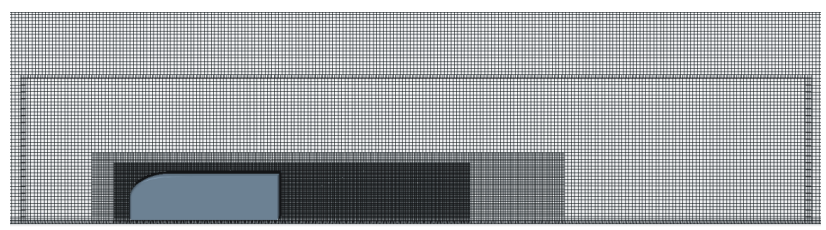

(a) Side view

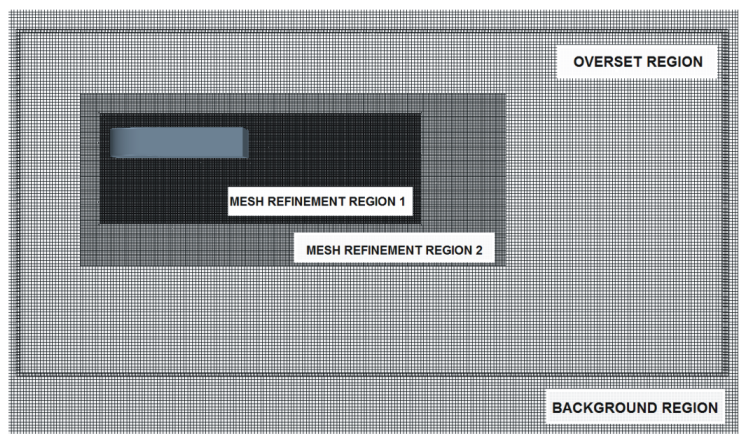

(b) Top view

Figure 8: Representation of mesh structure around the GTS vehicle model and overset mesh.

In the two-way coupled simulation and the one-way coupled simulation with moving vehicle, movement of the vehicle in the computational flow domain is enabled with the aid of overset mesh technique. Therefore the constructed mesh consist of two regions, i.e. an overlap region and a background region as presented in Fig. 8. There is an intermediate region between the two regions at which the solution field is interpolated between them. The overlap region including the vehicle performs its motion in the background 
region. The background mesh has a size of $22.6 \times 4.9 \times 1.4$ vehicle lengths, while the overlap region has a size of $5.3 \times 2.6 \times 1$ vehicle lengths. The meshes consist of unstructured hexahedral cells whose number is approximately $22 \times 10^{6}$ of which $9 \times 10^{6}$ are employed in the overlap region. On the other hand, flow domain the one-way coupled with fixed vehicle simulation has a size of $15.7 \times 4.9 \times 1.4$ vehicle lengths. Overset mesh is not employed in the one-way coupled with fixed vehicle simulation because GTS is fixed. Therefore, the number of the computational cells employed in this simulation becomes approximately $14 \times 10^{6}$. The time step used in all simulations is $6 \times 10^{-5}$ seconds.

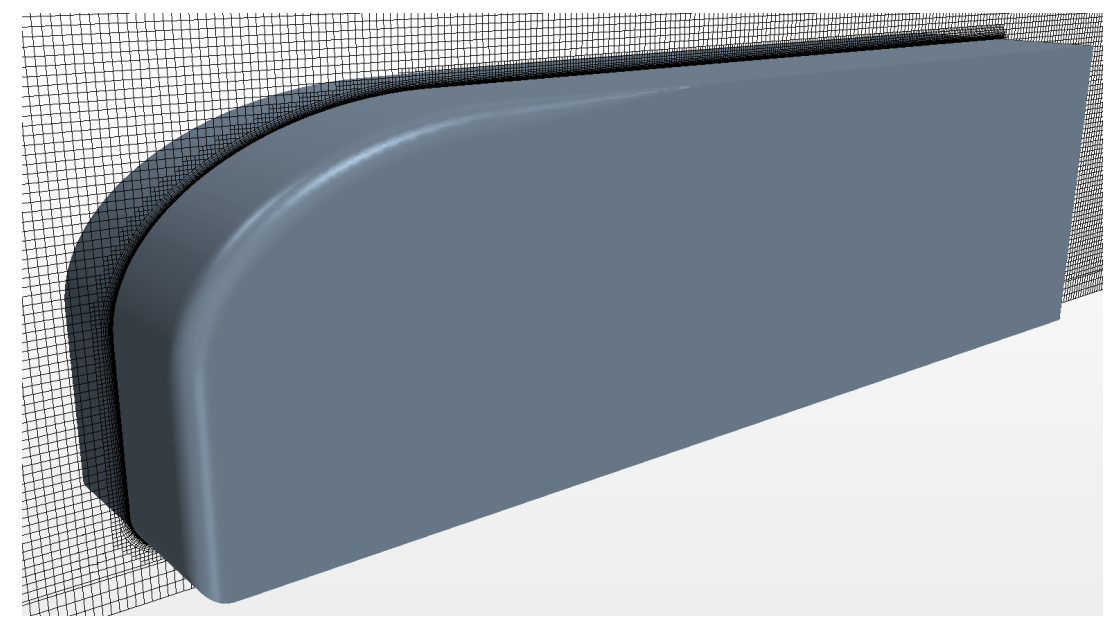

Figure 9: Mesh structures around the GTS.

In all simulations, two refinement regions are applied around the GTS. For the resolution of the boundary layer around the GTS, 20 prism layers with a total thickness in the wall normal direction of $0.0141 \mathrm{~m}$ with a stretching of 1.25 and a length of $0.0044 \mathrm{~m}$ are applied as presented in Fig. 9. The value of dimensionless wall distance $y^{+}$is less than 15 for the conditions used. 
Properties and size of the cells in the mesh regions are chosen based on the work of Winkler et al. (2016) which is also conducted to investigate effects of crosswind on the GTS.

Table 2: Drag coefficients.

\begin{tabular}{lccc}
\hline & Method & $R e_{w}$ & $C_{D}$ \\
\hline Croll et al. (1996) & Experimental & $1.6 \times 10^{6}$ & 0.247 \\
Storms et al. (2001) & Experimental & $1.6 \times 10^{6}$ & 0.249 \\
Unaune et al. (2005) & Numerical & $2 \times 10^{6}$ & 0.253 \\
The present study & Numerical & $2 \times 10^{6}$ & 0.251 \\
\hline
\end{tabular}

Comparison between the drag coefficient $C_{D}$ of the GTS obtained in the present study for the case of no crosswind with other drag coefficient results obtained in the previous literature for the same geometry is presented in Table 2. Because drag coefficient result obtained in the present study is close enough to both experimental and numerical results obtained in the previous literature, the mesh is assumed as appropriate and no further refinement is conducted.

Because a $1 / 8$ th scaled version of the GTS is employed in aerodynamics simulation, force and moment results, i.e. lateral force, yaw moment and roll moment, obtained in aerodynamics simulation should be scaled before using in the vehicle dynamics simulation. Scaling is conducted by assuming there is a dynamic similarity between the $1 / 8$ scaled GTS model employed in aerodynamics simulation and full scale GTS prototype employed in vehicle dynamics simulation. Therefore, force and moments are scaled according to definition of the force and the moment coefficients as follows. 


$$
\begin{gathered}
F_{\text {ya,prototype }}=F_{\text {ya,model }} \frac{u_{\infty, \text { prototype }}^{2}}{u_{\infty, \text { model }}^{2}} \frac{A_{\text {prototype }}}{A_{\text {model }}} \\
M_{z a, \text { prototype }}=M_{z a, \text { model }} \frac{u_{\infty, \text { prototype }}^{2}}{u_{\infty, \text { model }}^{2}} \frac{A_{\text {prototype }}}{A_{\text {model }}} \frac{L_{\text {prototype }}}{L_{\text {model }}} \\
M_{x a, \text { prototype }}=M_{x a, \text { model }} \frac{u_{\infty, \text { prototype }}^{2}}{u_{\infty, \text { model }}^{2}} \frac{A_{\text {prototype }}}{A_{\text {model }}} \frac{L_{\text {prototype }}}{L_{\text {model }}}
\end{gathered}
$$

251

Here, $F_{y a}, M_{z a}$ and $M_{x a}$ denote lateral force, yaw moment and roll moment, respectively. The free stream velocity or the velocity of the GTS is denoted by $u_{\infty}$. The cross-sectional area and length of the GTS are denoted by $A$ and $L$ respectively. The subscript prototype represents full scale GTS parameters and subscript model represents scaled parameters of the GTS.

\subsection{Vehicle Dynamics}

A single-track model with roll component is used to solve the vehicle dynamics equations. The state space representation of the vehicle dynamics equations are as follows (Lee et al., 2013).

$$
\begin{gathered}
x=\left[\begin{array}{llll}
v & \dot{\psi} & \dot{\phi} & \phi
\end{array}\right]^{T} \\
\dot{x}=A x+B
\end{gathered}
$$


$260 \quad A=\left[\begin{array}{cccc}-\frac{\lambda I_{x e q}}{m u I_{x x}} & \frac{\tau I_{x e q}}{m u I_{x x}}-u & -\frac{h C_{\phi}}{I_{x x}} & \frac{h\left(m g h-K_{\phi}\right)}{I_{x x}} \\ \frac{\tau}{u I_{z z}} & -\frac{\sigma}{u I_{z z}} & 0 & 0 \\ -\frac{\lambda h}{u I_{x x}} & \frac{\tau h}{u I_{x x}} & -\frac{C_{\phi}}{I_{x x}} & \frac{m g h-K_{\phi}}{I_{x x}} \\ 0 & 0 & 1 & 0\end{array}\right], B=\left[\begin{array}{c}-\frac{F_{y a}}{m} \\ -\frac{M_{z a}}{I_{z z}} \\ \frac{M_{x a}}{I_{x x}} \\ 0\end{array}\right]$

261 Definition of parameters in eqs. (6) and (7) are given in eqs. (8) to (11)

$$
\begin{gathered}
\lambda=C_{y 12}+C_{y 34} \\
\tau=C_{y 34} b-C_{y 12} f \\
\sigma=C_{y 12} f^{2}+C_{y 34} b^{2} \\
I_{x e q}=I_{x x}+m h^{2}
\end{gathered}
$$

262 Corresponding values of the parameters in eqs. (6) to (11) can be found 263 in Table 3.
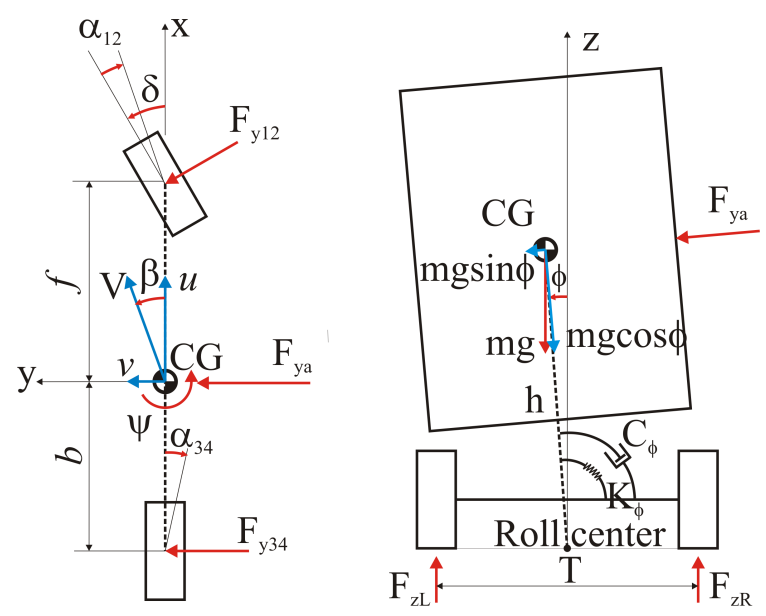

Figure 10: Single track model with roll degree of freedom. 
Table 3: Geometrical and mass properties of the GTS model employed in the study.

\begin{tabular}{llll}
\hline Vehicle's Parameter & Symbol & Data & Unit \\
\hline Length & $L$ & 12.2 & {$[\mathrm{~m}]$} \\
Height & $h$ & 3.6 & {$[\mathrm{~m}]$} \\
Width & $w$ & 2.6 & {$[\mathrm{~m}]$} \\
Track width & $\mathrm{T}$ & 2.25 & {$[\mathrm{~m}]$} \\
Length of the wheel base & $L_{w b}$ & 5.9 & {$[\mathrm{~m}]$} \\
Distance between front axle to CG & $f$ & 3.7 & {$[\mathrm{~m}]$} \\
Distance between rear axle to CG & $b$ & 2.2 & {$[\mathrm{~m}]$} \\
Mass & $m$ & 13,650 & {$[\mathrm{~kg}]$} \\
Linear tire stiffness for front tire & $\mathrm{C}_{y 12}$ & 250 & {$[\mathrm{kN} / \mathrm{rad}]$} \\
Linear tire stiffness for rear tire & $\mathrm{C}_{y 34}$ & 450 & {$[\mathrm{kN} / \mathrm{rad}]$} \\
Roll damping of suspension & $\mathrm{C}_{\phi}$ & 100 & {$[\mathrm{kN} / \mathrm{rad}]$} \\
Roll stiffness of suspension & $\mathrm{K}_{\phi}$ & 1,000 & {$[\mathrm{kNm} / \mathrm{rad}]$} \\
Yaw moment of inertia & $\mathrm{I}_{z z}$ & 200,000 & {$\left[\mathrm{kgm}{ }^{2} / \mathrm{rad}\right]$} \\
Roll moment of inertia & $\mathrm{I}_{x x}$ & 30,000 & {$\left[\mathrm{kgm}^{2} / \mathrm{rad}\right]$} \\
\hline
\end{tabular}

The coupled computations are performed by using high performance computing system at the PDC Center for High Performance Computing which is hosted at KTH Royal Institute of Technology. The HPC system run on a Cray XC40, based on Intel Haswell and Broadwell processors and Cray Aries interconnect technology. In the present study, in general, 24 nodes with a total of 768 cores are employed.

\section{Results and Discussion}

In this section, unsteady dynamic characteristics of the heavy vehicle model GTS which is subjected to crosswind gust are presented and discussed by using different coupling methods between aerodynamics and vehicle dy- 


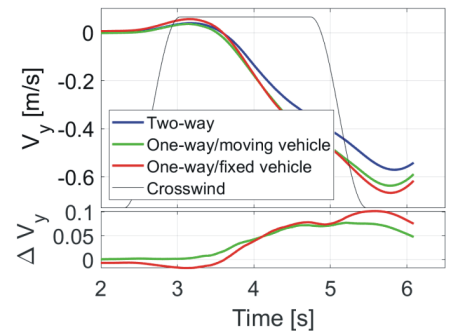

(a) Lateral velocity

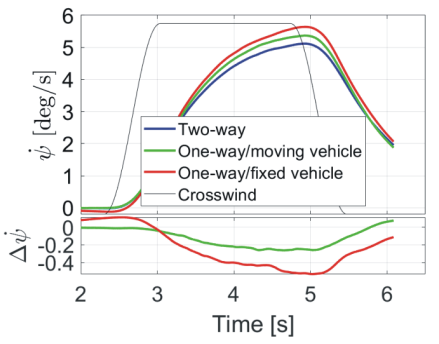

(b) Yaw rate

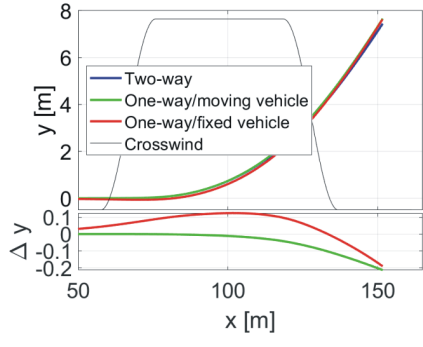

(c) Lateral displacement

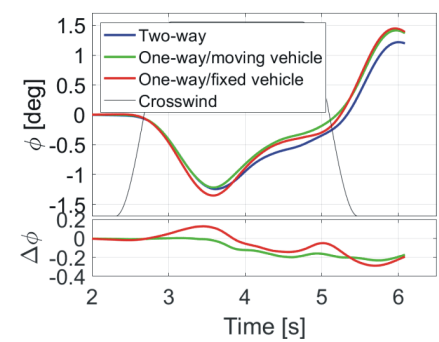

(d) Roll angle

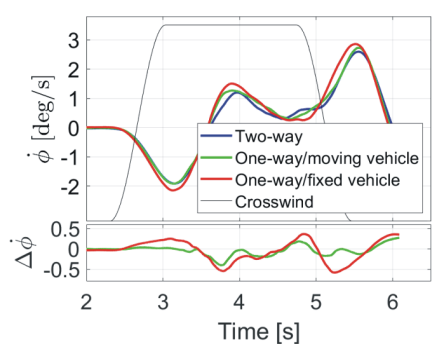

(e) Roll rate

Figure 11: Change of (a) lateral velocity $V_{y}$, (b) yaw rate $\dot{\psi}$, (c) lateral displacement $y$, (d) roll angle $\phi$, and (e) roll rate $\dot{\phi}$, with different coupling methods.

\subsection{Computational Efficiency of the Coupling Methods}

The computational efficiency of the coupling methods can be evaluated in two perspectives, i.e. the computation time and the complexity of the computational setup needed.

The computation times of two-way and one-way coupling methods considered in the present study are given in Table 4 . The differences between the 
Table 4: Computation times of each simulation.

\begin{tabular}{lc}
\hline Name of the coupling method & Computation time \\
\hline Two-way coupled simulation & $7: 10: 29$ \\
One-way coupled simulation with moving vehicle & $5: 54: 07$ \\
One-way coupled simulation with fixed vehicle & $6: 51: 50$ \\
\hline
\end{tabular}

computation times of the coupling methods are not large. The results of the two-way coupled simulation and one-way coupled simulation with moving vehicle show clear identification of the computation time difference between two methods because all settings of the simulations are the same except the way of coupling between aerodynamics and vehicle dynamics equations. However, although the one-way coupled simulation with fixed vehicle has low number of computational cells, its computation time is almost the same as the computation time of the two-way coupled simulation. The reason for this result is that the one-way coupled simulation with fixed vehicle needs more time step in order to prevent disturbed flow upstream of the GTS due to usage of moving boundary condition to simulate crosswind.

On the other hand, the complexity of the computational setup needed, which is the other factor in evaluating computational efficiency of the coupling methods, is lower in the one-way coupled simulation with fixed vehicle because there is no need to a computational setup for the communication between the simulations in every time steps and there is no need to use overset mesh in the aerodynamics simulation.

As a result, whereas the one-way coupling methods have no considerable advantage over the two-way coupling method in terms of computation 
time, complexity in their computational setup is less than two-way coupling method.

\subsection{Vehicle Dynamics}

Results related to vehicle dynamics are presented in Fig. 11 by comparing three different simulations. In the figures, differences between the results of different coupling methods are directly given in a subplot. Crosswind profile is also plotted on each figure in order to better assessment of its effects on the dynamic characteristics of the GTS.

The results presented in Fig. 11 reveal that as soon as the GTS is subjected to the crosswind, its yaw rate $\dot{\psi}$, increases. Because of increase in its yaw rate, its lateral velocity $V_{y}$, starts to increase in negative y-direction immediately after it experiences a slight increase in positive y-direction. Similarly, roll angle $\phi$, increases in negative direction, i.e. counter-clockwise direction, after being affected by the crosswind. Whereas the maximum values of the lateral velocity and the yaw rate are obtained at the end of the crosswind, the maximum value of the roll angle is obtained nearly at the midlocation of the crosswind. Finally, GTS starts to exhibit lateral displacement $y$, in the same direction with the application of the crosswind.

As presented in Fig. 11, results of the one-way coupled simulations have good harmony with results of the two-way coupled simulation until $t=3.0 \mathrm{~s}$, i.e. at the beginning of the crosswind. However, this good harmony between the results can not be kept further due to not responding of the one-way coupled simulations to highly unsteady effects of the crosswind. The results start to differ with each other, especially results of the lateral velocity and the yaw rate, after further movement of the GTS into the crosswind. In 
general, the one-way coupled simulations overestimate the results. Absolute value of the differences between the results normalized by the absolute maximum value of the results obtained by two-way coupled simulation are less than $20 \%$ in the results of lateral velocity, yaw rate, roll angle and roll rate. On the other hand, corresponding differences in lateral displacement between the results are relatively lower than the others, i.e. they are less than $3 \%$. Furthermore, the maximum difference in lateral displacement of the vehicle is obtained between the two-way coupled simulation and the one-way coupled simulation with fixed vehicle, i.e. $\Delta y_{\max }=0.2 \mathrm{~m}$. In the studies of Winkler et al. (2016), Carbonne et al. (2016) and Forbes et al. (2016), one-way coupled simulations also overestimate lateral displacement of the vehicle at slightly different levels. Winkler et al. (2016) find it as 3.9\%, Carbonne et al. (2016) find it as maximum $1 \%$ and Forbes et al. (2016) find it close to zero. Differences in the results might be caused by using different vehicle models and different equations in their studies. For example, in the study of Winkler et al. (2016), roll motion of the vehicle is not considered in the simulations and in the studies of Carbonne et al. (2016) and Forbes et al. (2016), passenger car models are employed as the vehicle model.

The results of the one-way coupled with moving vehicle simulation (employing overset mesh) are in general closer to the results of two-way coupled simulation than the results of one-way coupled simulation with fixed vehicle. Therefore, it can be concluded that simulation of the flow field by employing moving vehicle (overset mesh) rather than employing a fixed vehicle can improve obtained results. As a conclusion, there are differences between results of the one-way coupled simulations and the two-way coupled simulation at a 


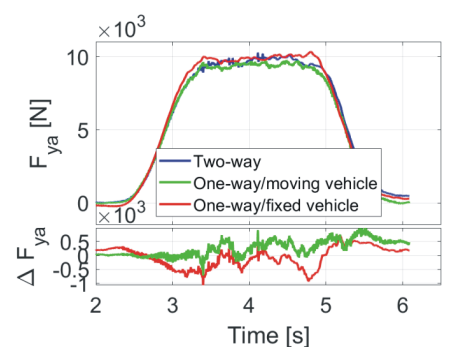

(a) Lateral force

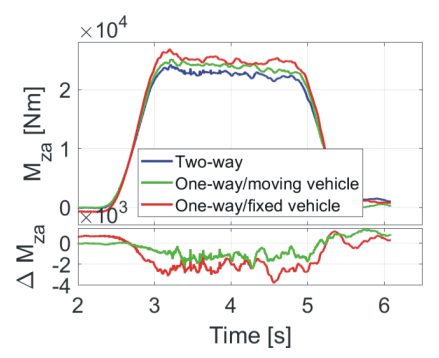

(b) Yaw moment

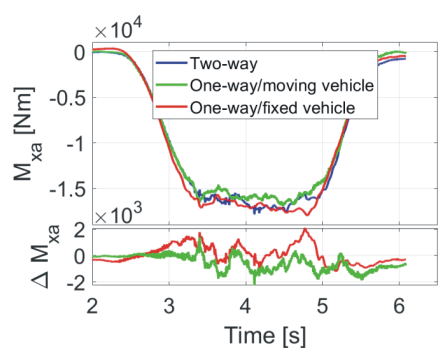

(c) Roll moment

Figure 12: Change of (a) lateral force $F_{y a}$, (b) yaw moment $M_{z a}$, and (c) roll moment $M_{x a}$ with different coupling methods.

\subsection{Aerodynamics}

\subsubsection{Force and Moment Results}

Comparison of the computed aerodynamic force and moments exerted on the GTS by oncoming crosswind are given in Fig. 12. Because, in the present study, the main focus is on lateral, yaw and roll dynamics of the vehicle, only the results of the lateral force $F_{y a}$, the roll moment $M_{x a}$ and the yaw moment $M_{z a}$, are presented in the figure. In line with the results given in Fig. 11, the one-way coupled with fixed vehicle simulation overestimate force and moment results, especially the yaw moment. Absolute value of the differences between the results normalized by the maximum value of the results obtained in the two-way coupled simulation are less than 20 percent in all results presented in Fig. 12.

In order to understand reasons for the differences in the force and moment results given in Fig. 12, change of the force and moment coefficients, i.e. $C_{\text {side }}, C_{\text {yaw }}$ and $C_{\text {roll }}$, on each surface of the GTS with time are investigated 


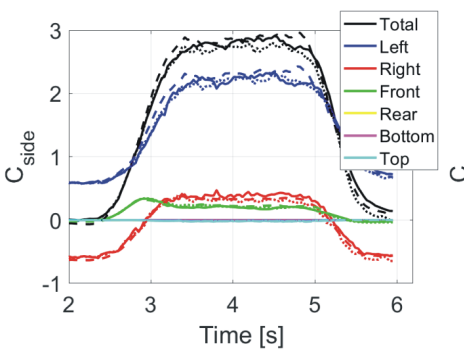

(a) Lateral force coefficient

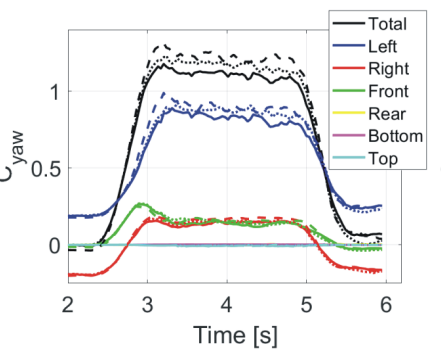

(b) Yaw moment coefficient

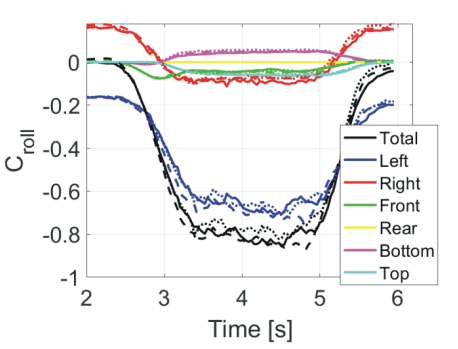

(c) Roll moment coefficient

Figure 13: Comparison of (a) the lateral force coefficient $C_{\text {side }}$, (b) the yaw moment coefficient $C_{\text {yaw }}$ and (c) the roll moment coefficient $C_{\text {roll }}$, obtained by different coupling methods on each surface of the GTS (—represents two-way coupled simulation, …...represents one-way coupled with moving vehicle simulation, - - -represents one-way coupled with fixed vehicle simulation).

in Fig. 13. It can be explicitly understood from Fig. 13 that the front and the lateral surfaces have the main contribution to the lateral force, the yaw and the roll moments. Besides, contribution of the rear, the bottom and the top surfaces of the GTS to the corresponding force and the moments are little. The only exception is that the bottom and the top surfaces have considerable contribution to the roll moment of the GTS in opposite direction in which they cancel each other as a result. Before the crosswind starts, i.e. before $t=2.67 \mathrm{~s}$, total force and moment coefficient results obtained by using the one-way coupled with fixed vehicle simulation show some differences from results of the other simulations. That means, upstream flow field created by the crosswind in the one-way coupled with fixed vehicle simulation produces some lateral force, yaw and roll moments on the GTS before it hits on the GTS. However, as soon as the crosswind starts, results of all simulations overlap each other for a while. Then, they start to differ from each other while the GTS is fully immersed in the crosswind. According to the results presented in Fig. 13, differences on the left (leeward) surface of the GTS are 
the main source of the differences between all force and moment results.

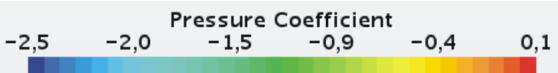

Two-way coupled simulation
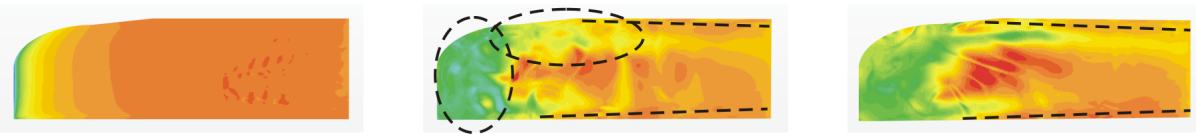

One-way coupled simulation with moving vehicle
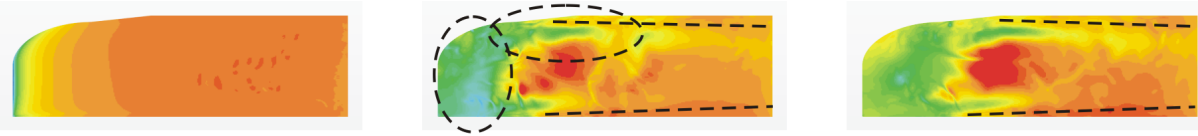

One-way coupled simulation with fixed vehicle

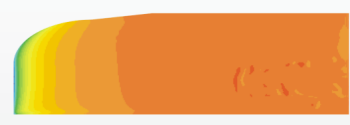

$\mathrm{t}=2.67 \mathrm{~s}$

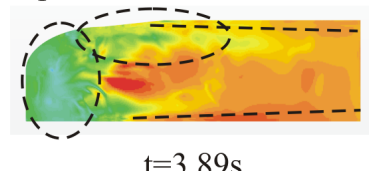

$\mathrm{t}=3.89 \mathrm{~s}$

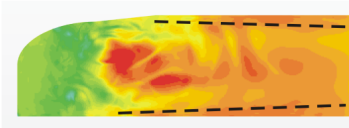

$\mathrm{t}=5.11 \mathrm{~s}$

Figure 14: The pressure coefficient $C_{p}$, on the left (leeward) lateral surface of the GTS obtained by using three different coupling methods.

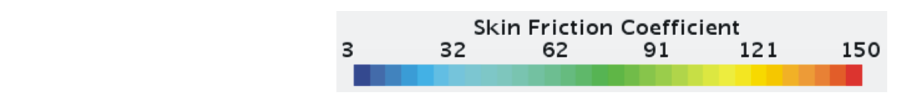

Two-way coupled simulation
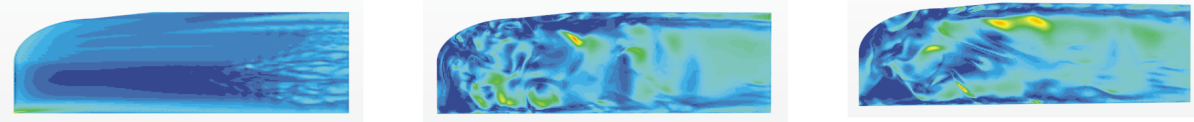

One-way coupled simulation with moving vehicle
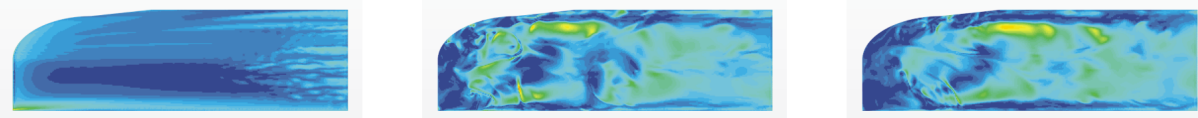

One-way coupled simulation with fixed vehicle

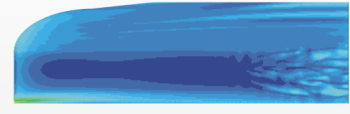

$\mathrm{t}=2.67 \mathrm{~s}$

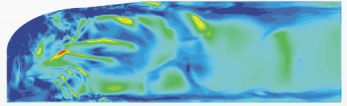

$\mathrm{t}=3.89 \mathrm{~s}$

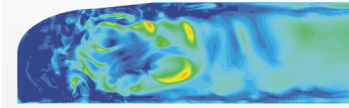

$\mathrm{t}=5.11 \mathrm{~s}$

Figure 15: Skin friction coefficient $C_{f}$, on the left (leeward) lateral surface of the GTS obtained by using three different coupling methods.

Therefore, in Figs. 14 and 15, the pressure coefficient $C_{p}$, and skin friction coefficient $C_{f}$, results obtained in all simulations at three different instants, 
e.g. $t=2.67 \mathrm{~s}, 3.89 \mathrm{~s}, 5.11 \mathrm{~s}$, are investigated. At $t=2.67 \mathrm{~s}$, because the crosswind has just started, patterns of pressure coefficient contours are almost identical. However, after fully immersing of the GTS into the crosswind, i.e. $t=3.89$ and $5.11 \mathrm{~s}$, a massive reduction of the pressure coefficient which is resulted in a wake region on the front part of the left (leeward) lateral surface of the GTS is observed. Accordingly, the main difference between the results of the two-way and the one-way couplings is observed at $t=3.89 \mathrm{~s}$ on the front part of the left lateral surface as presented by dashed ellipses in Fig 14. Additionally, traces of longitudinal vortices emerged from upper and lower part at the front of the GTS on the left lateral surface are indicated with dashed straight lines on each figures. Skin friction coefficient results, given in Fig. 15, present starting location of the upper and the lower longitudinal vortices at $t=2.67 \mathrm{~s}$ in Fig. 15. At this instant, prediction of all simulations are rather close to each other since the GTS has a bluff body like geometry. Between these upper and lower longitudinal vortices, high pressure and skin friction coefficient region is observed in Figs. 14 and 15. This region corresponds to traces of hairpin vortices emerged from vertical front edge of the left (leeward) lateral surface of the GTS.

Pressure and skin friction coefficient results given on top surface of the GTS in Figs. 16 and 17 explain formation mechanism of the vortices further. At the beginning of the crosswind, i.e. $t=2.67 \mathrm{~s}$, pressure and skin friction coefficients show almost uniform change from the leading edge. However, as the GTS is fully immersed in the crosswind, a low pressure region appears on the top surface which shows trace of the vortex emerging on the right (windward) front edge and then crossing the top surface from right to left. 


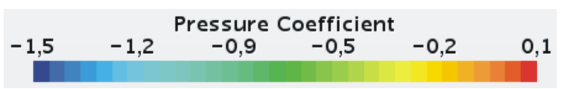

Two-way coupled simulation
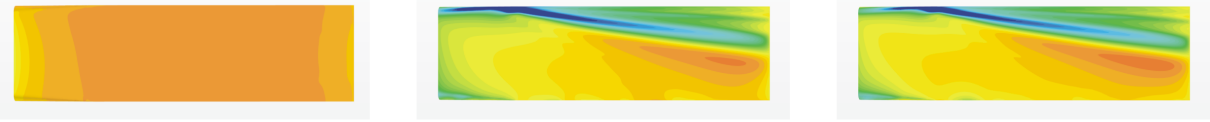

One-way coupled simulation with moving vehicle

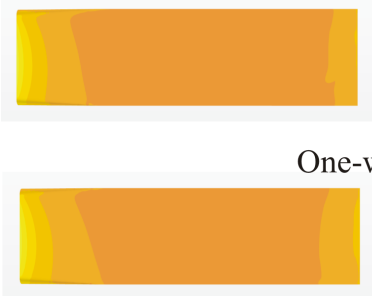

$\mathrm{t}=2.67 \mathrm{~s}$
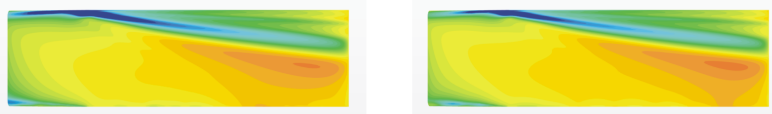

One-way coupled simulation with fixed vehicle

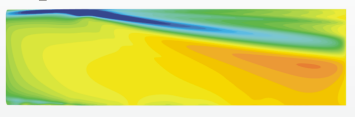

$\mathrm{t}=3.89 \mathrm{~s}$

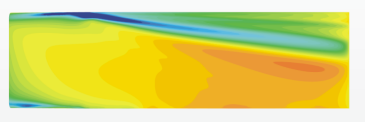

$\mathrm{t}=5.11 \mathrm{~s}$

Figure 16: Pressure coefficient $C_{p}$ on top surface of the GTS with different coupling methods.

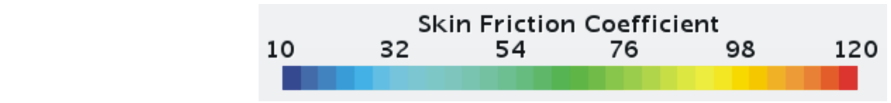

Two-way coupled simulation
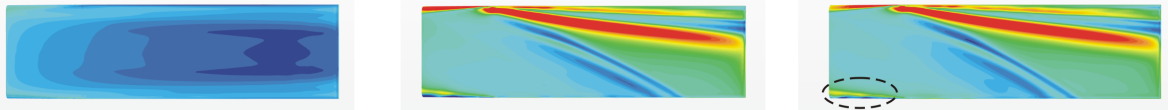

One-way coupled simulation with moving vehicle
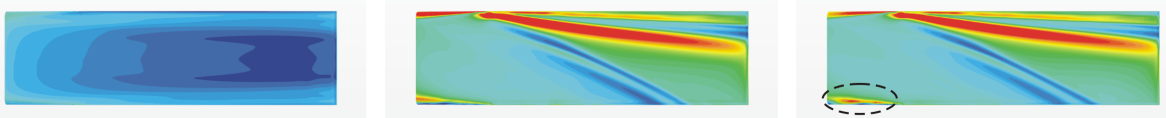

One-way coupled simulation with fixed vehicle

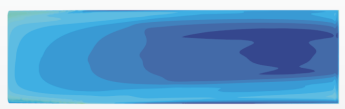

$\mathrm{t}=2.67 \mathrm{~s}$

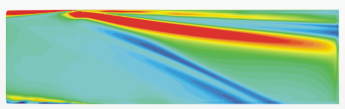

$\mathrm{t}=3.89 \mathrm{~s}$

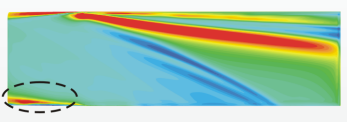

$\mathrm{t}=5.11 \mathrm{~s}$

Figure 17: Skin friction coefficient $C_{f}$ on top surface of the GTS with different coupling methods.

As for the differences between the results on top surface of the GTS, oneway coupled simulations overestimate skin friction coefficient results at front

left (leeward) part of the top surface at which secondary longitudinal vortex 
emerge and interact other vortices on the left (leeward) surface of the GTS. Three dimensional interaction of theses vortices are presented in Fig. 22 and their detailed investigations are given in the next section.

\subsubsection{Velocity Field Results}

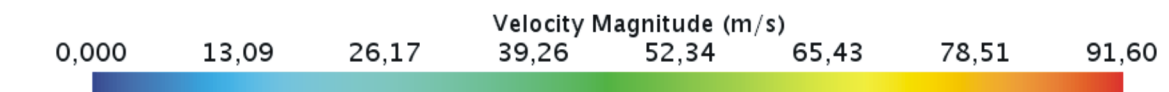

Two-way coupled simulation
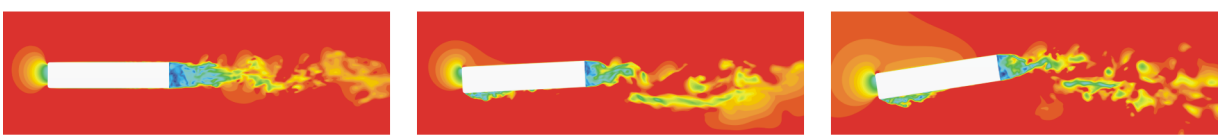

One-way coupled simulation with moving vehicle
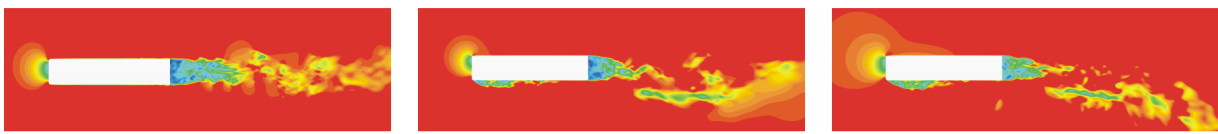

One-way coupled simulation with fixed vehicle

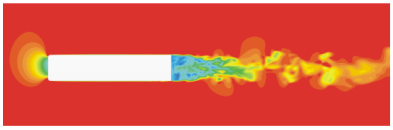

(a) $\mathrm{t}=2.67 \mathrm{~s}$

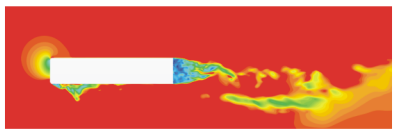

(b) $\mathrm{t}=3.89 \mathrm{~s}$

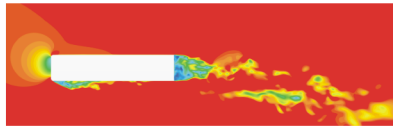

(c) $\mathrm{t}=5.11 \mathrm{~s}$

Figure 18: Contours of unsteady velocity magnitude $\mathrm{U}$, at three different instants in the horizontal xz-plane at mid-height of the GTS, e.g. (a) $t=2.67 \mathrm{~s}$ starting point of the crosswind, (b) $t=3.89 \mathrm{~s}$ mid-point of the crosswind, (c) $t=5.11 \mathrm{~s}$ end-point of the crosswind.$$
27
$$

8 9

Contour plots of unsteady velocity magnitudes obtained from three different simulations are given in Fig. 18 at three different instants. At $t=2.67$ $\mathrm{s}$, because the GTS has just begun to be affected by the crosswind, the velocity field around it looks similar to a characteristic one which is encountered around bluff bodies. It means that there are a stagnation flow region in the front and a wake flow region in the rear part of the GTS. At $t=3.89 \mathrm{~s}$, which is at the mid point of the crosswind, the velocity field around the GTS distorts 
and new flow regions start to appear due to yawing effect of the incoming crosswind flow. For example, on the left (leeward) side of the GTS, a separated flow region appear on the upstream part of the left (leeward) lateral surface and a secondary wake region appear in the wake flow downstream of the GTS. Besides, the stagnation region in the front part of the GTS shifts to right (windward) side of the front surface. Main differences observed at this instant between the results of the one-way and the two-way coupled simulations are dimensions of the stagnation region at the front part of the GTS. Two-way coupled simulation has the largest stagnation flow region because it rotates in positive yaw direction (counter clockwise) in response to the effects of the crosswind. At $t=5.11 \mathrm{~s}$, which is at the end point of the crosswind, flow features appearing in the previous instant keep their existence with enlarged dimensions.

Results of turbulent kinetic energy TKE, presented in Fig. 19 reveal the amount of the turbulence mixing around the GTS caused by the crosswind. TKE is directly related to the dynamics of the flow. In general, results of all simulations have the same patterns of TKE. Namely, high values of TKE are predicted in the shear layers developed around the GTS. Then, they dissipate gradually in the downstream flow direction. As it is expected, at the start of the crosswind i.e. $t=2.67 \mathrm{~s}$, TKE is only high along the right and left shear layers emerged from lateral surfaces in the near wake region downstream of the GTS. However, after fully immersing of the GTS in the crosswind, i.e. at $t=3.89 \mathrm{~s}$ and $5.11 \mathrm{~s}$, an additional region of high TKE appears on the front part of the left (leeward) lateral surface. Besides, concentrations of TKE contours increase on right (windward) shear layer in the near wake region 


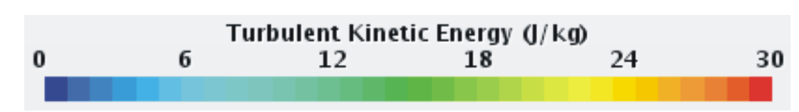

Two-way coupled simulation
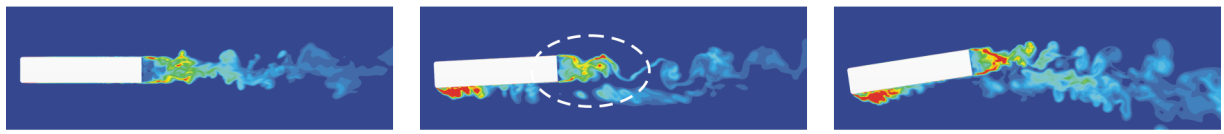

One-way coupled simulation with moving vehicle
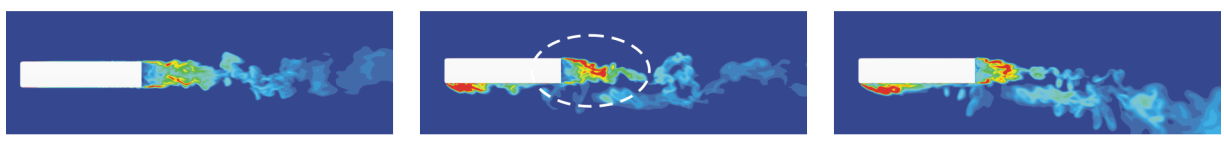

One-way coupled simulation with fixed vehicle

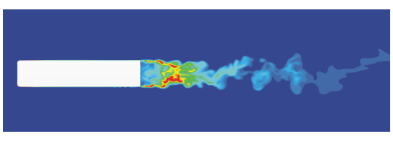

(a) $\mathrm{t}=2.67 \mathrm{~s}$

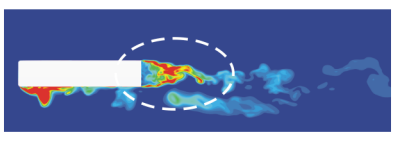

(b) $\mathrm{t}=3.89 \mathrm{~s}$

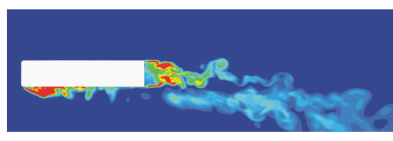

(c) $\mathrm{t}=5.11 \mathrm{~s}$

Figure 19: Contours of turbulent kinetic energy TKE, at three different instants in the horizontal xz-plane at mid-height of the GTS, e.g. (a) $t=2.67 \mathrm{~s}$ starting point of the crosswind, (b) $t=3.89 \mathrm{~s}$ mid-point of the crosswind, (c) $t=5.11 \mathrm{~s}$ end-point of the crosswind.

downstream of the GTS at $t=3.89 \mathrm{~s}$. This increase is more pronounced in the results of the one-way coupled simulations than the two-way coupled simulation.

In Fig. 20, contours of unsteady vorticity $\omega_{y}$, are presented at three different instants in the horizontal xz-plane at mid-height of the GTS. In this figure, blue and red colors correspond clockwise and counter clockwise rotation of the vorticity concentrations respectively. The most noticeable difference between the results at $t=2.67 \mathrm{~s}$ is in shedding frequency of the vorticity concentrations formed in the wake region downstream of the GTS. Comparing with the fixed vehicle simulation, longer shedding period of the vorticity concentrations is observed in the wake region of the simulations 

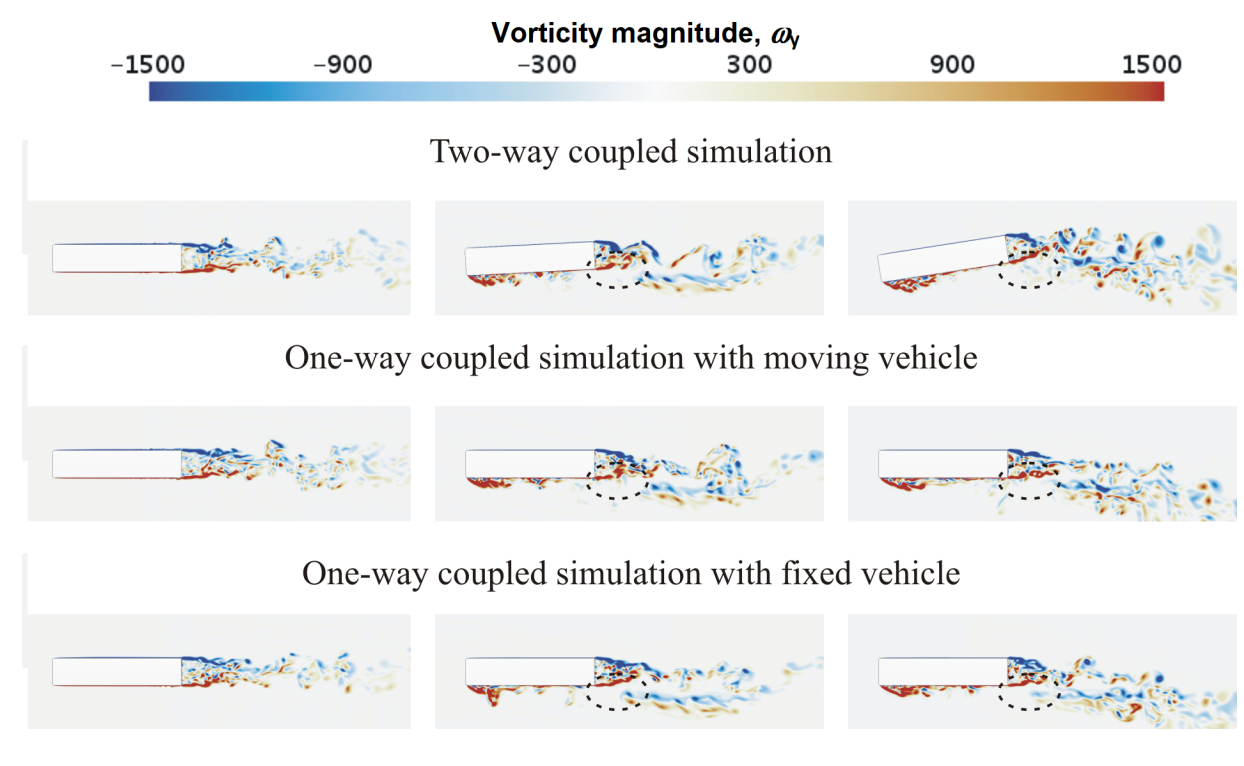
(a) $\mathrm{t}=2.67 \mathrm{~s}$
(b) $\mathrm{t}=3.89 \mathrm{~s}$
(c) $\mathrm{t}=5.11 \mathrm{~s}$

Figure 20: Contours of vorticity $\omega_{y}$, at three different instants in the horizontal xz-plane at mid-height of the GTS, e.g. (a) $t=2.67 \mathrm{~s}$ starting point of the crosswind, (b) $t=3.89$ $\mathrm{s}$ mid-point of the crosswind and (c) $t=5.11 \mathrm{~s}$ end-point of the crosswind.

employing moving vehicle, i.e. overset mesh. At $t=3.89 \mathrm{~s}$, because the vehicle is completely immersed into the crosswind, inline with the above mentioned results, a secondary wake region appears at front part of the left (leeward) lateral surface of the GTS in all simulations. Besides, an additional couple of vorticity concentrations appear on the left (leeward) side of the wake region in the downstream part of the GTS. This secondary couple of vorticity concentrations interact with main couple of vorticity concentrations in the wake region downstream of the GTS as presented in 20 at $t=3.89 \mathrm{~s}$ and $t=5.11 \mathrm{~s}$. Starting location of this secondary vorticity concentration couple is closer to the GTS in the one-way coupled with fixed vehicle simulation than the corresponding starting location of the secondary vorticity concentrations 
477

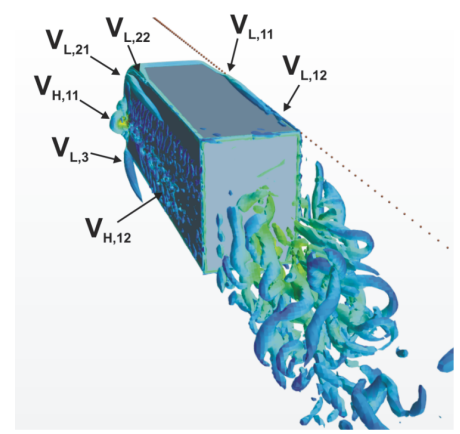

(a) Two-way

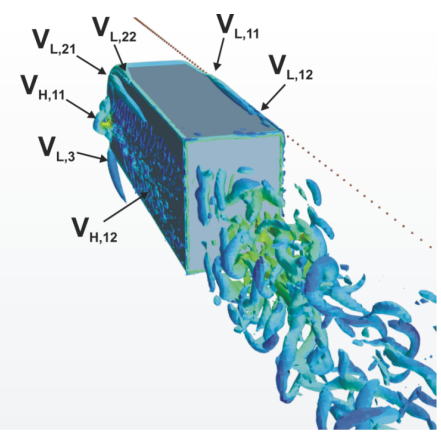

(b) One-way moving vehicle

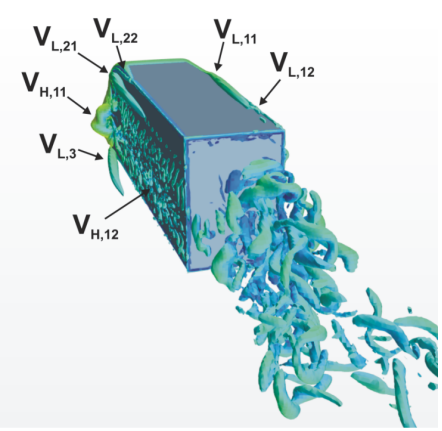

(c) One-way fixed vehicle

Figure 21: Formation of unsteady vortices around the GTS obtained from three different simulations at $t=3.0 \mathrm{~s}$ with the aid of $\mathrm{Q}$ criterion, $\mathrm{Q}=10^{6}$.

Formation of the vortices developing around the GTS, which are computed by using Q criterion, are presented in Fig. 21. This figure give information about starting location and characteristics of the vortices emerging from the front part of the GTS after being effected by the crosswind. There are longitudinal vortices which emerge from each upper and lower corners at the front part of the GTS, e.g. $\mathrm{V}_{\mathrm{L}, 11}, \mathrm{~V}_{\mathrm{L}, 21}, \mathrm{~V}_{\mathrm{L}, 3}$ as presented in Fig. 21. Besides them, there are secondary longitudinal vortices which are formed around upper edges connecting top and lateral surfaces, e.g. $\mathrm{V}_{\mathrm{L}, 12}$ and $\mathrm{V}_{\mathrm{L}, 22}$. These secondary vortices develop due to interaction between flow streams coming from neighbouring surfaces with the crosswind flow. In addition to the longitudinal vortices, there are hairpin vortices developing on the left (leeward) lateral surface, e.g. $\mathrm{V}_{\mathrm{H}, 11}$ and $\mathrm{V}_{\mathrm{H}, 12}$. These large scale hairpin vortices form at the front part with large scales and then they move on the left lateral surface in downstream direction with decreasing scales.

Finally, development of the above-mentioned vortices around the GTS 

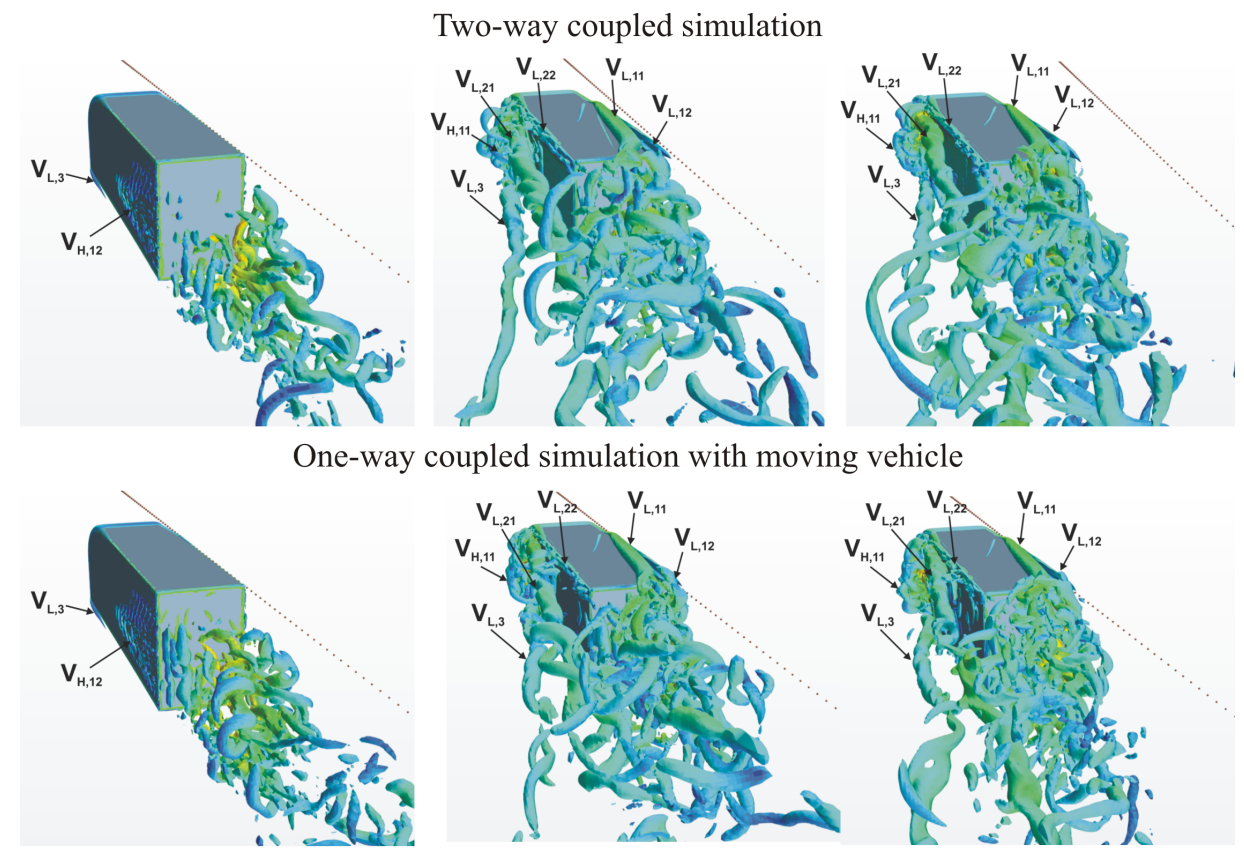

One-way coupled simulation with fixed vehicle

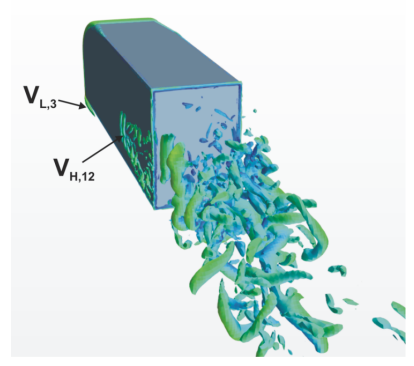

(a) $\mathrm{t}=2.67 \mathrm{~s}$

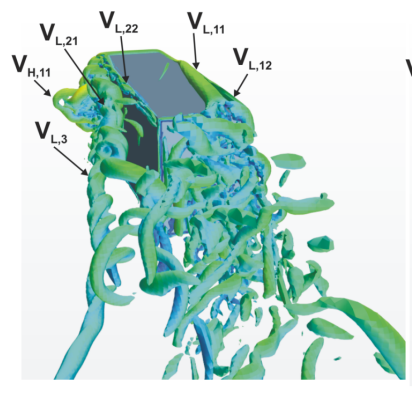

(b) $\mathrm{t}=3.89 \mathrm{~s}$

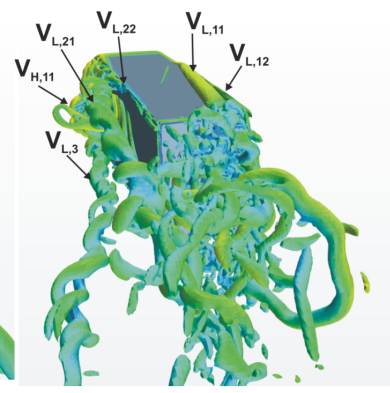

(c) $\mathrm{t}=5.11 \mathrm{~s}$

Figure 22: Development of vortices around the GTS at three different instants, e.g. (a) $t$ $=2.67 \mathrm{~s}$ starting point of the crosswind, (b) $t=3.89 \mathrm{~s}$ mid-point of the crosswind, (c) $t$ $=5.11 \mathrm{~s}$ end-point of the crosswind, with the aid of $\mathrm{Q}$ criterion, $\mathrm{Q}=10^{6}$.

from beginning to end of the crosswind event are presented in Fig. 22. At the beginning of the crosswind i.e. $t=2.67 \mathrm{~s}$, besides vortex structures observed in the wake downstream of the GTS, the longitudinal vortex emerging from bottom front part of the left (leeward) lateral surface and hairpin vortices on the rear part of the left lateral surface are observed. However, as the 
GTS fully immerse in the crosswind, all vortices develop more and interact with each other resulting in a more complicated flow field around the GTS. Hairpin vortices $\mathrm{V}_{\mathrm{H}, 11}$, disturb the longitudinal vortices $\mathrm{V}_{\mathrm{L}, 21}$ and $\mathrm{V}_{\mathrm{L}, 3}$ to make them unstabilized. Besides, secondary longitudinal vortices $\mathrm{V}_{\mathrm{L}, 12}$ and $\mathrm{V}_{\mathrm{L}, 22}$ interact with other longitudinal vortices $\mathrm{V}_{\mathrm{L}, 11}$ and $\mathrm{V}_{\mathrm{L}, 21}$, respectively. While, as soon as longitudinal vortex $\mathrm{V}_{\mathrm{L}, 11}$ reaches to the rear end of the GTS, it breaks down into the wake region, the other longitudinal vortices $\mathrm{V}_{\mathrm{L}, 21}$ and $\mathrm{V}_{\mathrm{L}, 3}$ keeps their existence in the wake region. Therefore, Fig. 22 reveals the physical mechanism which produces secondary wake on the leeward side of the wake region downstream of the GTS. Interactions between the vortices $\mathrm{V}_{\mathrm{L}, 12}$ and $\mathrm{V}_{\mathrm{L}, 21}$ and flow stream coming from right (windward) side of the GTS generate the secondary wake region. Finally, when comparing obtained results between each other, one can observe that one-way coupled simulation with fixed vehicle estimates the strongest longitudinal vortices caused by crosswind which might also explains why one-way coupled with fixed vehicle simulation overestimates vehicle dynamics parameters presented in Fig. 11.

\section{Conclusion}

The aim of the present study is to understand effects of the coupling methods, e.g. one-way and two-way couplings, on the solution of the dynamic characteristics of heavy ground vehicles which are subjected to crosswind gusts. Therefore, the degree of the complexity required to obtain accurate solutions for industrial applications can be understood.

One-way coupled simulations, in general, overestimate the absolute maximum values of the vehicle dynamics results. The lateral velocity followed 
by the yaw rate present the highest differences between the results of the coupling methods. In lateral displacement results, the maximum difference between results of the two-way coupled simulation and one-way coupled with fixed vehicle simulation is $\Delta y_{\max }=0.2 \mathrm{~m}$. Because the vehicle dynamics results of the one-way coupled with moving vehicle simulation are, in general, closer to the results of the two-way coupled simulation than the results of the one-way coupled with fixed vehicle simulation, It can be concluded that employing moving vehicle (overset mesh) improve results obtained.

In aerodynamics results, in line with the vehicle dynamics results, the one-way coupled with fixed vehicle simulation overestimate force and moment results, especially the yaw moment. It is found that the front and the lateral surfaces have the main contribution to the lateral force, the yaw and the roll moments. The main difference in between the pressure and friction coefficient results of the simulations is observed at $t=3.89 \mathrm{~s}$ on the front part of the left lateral surface. Therefore it can be concluded that the flow field resolved by the simulations on the left (leeward) surface of the GTS has the biggest contribution to differences between force and moment results. Additionally, velocity fields in the near wake region downstream of the GTS reveal some more differences between the results of the simulations. For example, increase in concentrations of the TKE contours on the right (windward) shear layer in the near wake region at $t=3.89 \mathrm{~s}$, is more pronounced in the results of the one-way coupled simulations than the two-way coupled simulation. Additionally, location of the secondary vorticity concentration couple developed in the near wake region due to the crosswind is closer to the GTS in the case of one-way coupled with fixed vehicle simulation than 
the other simulations.

As a conclusion, two-way coupled simulations are necessary to compute effects of the crosswind on ground vehicles accurately, especially for cases in which high amount of yaw angles are expected. On the other hand, considering the differences between one-way and two-way coupled simulations, one-way coupled simulations might provide a good alternative at initial stages of design processes in industrial applications in which there is a lack of computational facility and accuracy is not a priority.

\section{Acknowledgment}

The authors would like to gratefully acknowledge the co-funding they have received for this work from the Swedish Innovation Agency Vinnova (grant number 2017-03391), the Centre for ECO2 Vehicle Design at KTH (itself funded by Vinnova grant number 2016-05195) and the strategic research area TRENoP. The computations were performed on resources provided by the Swedish National Infrastructure for Computing (SNIC) at the PDC Center for High Performance Computing (PDC-HPC).

\section{References}

Carbonne, L., Winkler, N., Efraimsson, G.: Use of Full Coupling of Aerodynamics and Vehicle Dynamics for Numerical Simulation of the Crosswind Stability of Ground Vehicles. SAE Int. J. Commer. Veh., 9(2), 359-370, 2016. doi:10.4271/2016-01-8148 
Cheli, F., Corradi, R., Sabbioni, E., Tomasini, G., 2011(a). Wind tunnel tests on heavy road vehicles: Crosswind induced loads - Part 1. J. Wind Eng. Ind. Aerodyn., 99, 1000-1010. doi:10.1016/j.jweia.2011.07.009

Cheli,F., Ripamonti, F., Sabbioni, E., Tomasini, G., 2011(b). Wind tunnel tests on heavy road vehicles: Crosswind induced loads - Part 2. J. Wind Eng. Ind. Aerodyn., 99, 1011-1024. doi:10.1016/j.jweia.2011.07.007

Choi, H., Lee, J., Park, H.: Aerodynamics of Heavy Vehicles. Annual Review of Fluid Mechanics, 2014. 46:441-68. doi:10.1146/annurev-fluid-011212140616

Croll, R.H., Gutierrez, W.T., Hassan, B., Suazo, J.E., Riggins, A.J.: Experimental Investigation of the Ground Transportation Systems (GTS) Project for Heavy Vehicle Drag Reduction. In: 1996 SAE International Congress and Exposition, Detroit, Michigan, February 26-29, 1996.

Favre, T., 2011. "Aerodynamics simulations of ground vehicles in unsteady crosswind", PhD thesis, KTH School of Engineering Sciences.

Forbes, D.C., Page, G.J., Passmore, M.A., Gaylard, A.P.: A Fully Coupled, 6Degree-of-Freedom, Aerodynamic and Vehicle Handling Crosswind Simulation using the DrivAer Model. SAE Int. J. Passeng. Cars - Mech. Syst., 9(2): 710-722 (2016). doi:10.4271/2016-01-1601

Ghuge, H.: Detached Eddy Simulations of a simplified Tractor-Trailer Geometry. MSc Thesis, Auburn, Alabama May 10, 2007.

Guilmineau, E., Chikhaoui, O., Deng, G.B., Visonneau, M., 2013. Crosswind 
effects on a simplified car model by a DES approach. Computers and Fluids, 78, 29-40.

Gutierrez, W.T., Hassan, B., Croll, R.H., Rutledge, W.H.: Aerodynamics overview of the ground transportation systems (GTS) project for heavy vehicle drag reduction. In: 1996 SAE International Congress and Exposition, Detroit, Michigan, February 26-29, 1996.

Huang, T., Gu, Z., Feng, C.: "Transient Aerodynamics simulations of a road vehicle in the crosswind condition coupled with the vehicle's motion". Proc IMechE Part D: J Automobile Engineering, 1-16. doi:10.1177/09.54407017704609

Huang, T.M., Gu, Z.Q., Feng, C.J.: Coupled Analysis of Unsteady Aerodynamics and Vehicle Motion of a Passenger Car in crosswind Condition. Journal of Applied Fluid Mechanics, 10(2), 625-637 (2017). doi:10.18869/acadpub.jafm.73.239.26639

Huang, T., Li, S., Wan, Z., Gu, C.: "Investigation of vehicle stability under crosswind conditions based on coupling methods". Proc IMechE Part D: J Automobile Engineering, 1-13. doi:10.1177/0954407018822424

Ishioka, H., Onishi, K., Nakasato, K., Nakashima, T., Tsubokura, M.: Coupled 6Dof Motion and Aerodynamics Simulation of Road Vehicles in Crosswind Gusts. In: 33rd AIAA Applied Aerodynamics Conference, AIAA AVIATION Forum 22-26 June 2015, American Institute of Aeronautics and Astronautics, Dallas, TX (2015). doi: 10.2514/6.2015-3308 
Lee, S., Kasahara, M., Mori, Y.: Roll Damping Control of a Heavy Vehicle under the Strong Crosswind. In: 7th IFAC Symposium on Advances in Automotive Control, 4-7 September 2013, 219-224 The International Federation of Automatic Control, Tokyo, Japan (2013). doi: $10.3182 / 20130904-4-J P-2042.00053$

Li, S., Gu, Z., Huang, T., Chen, Z., Liu, J.: "Coupled analysis of vehicle stability in crosswind on low adhesion road", International Journal of Numerical Methods for Heat \& Fluid Flow. doi:10.1108/HFF-01-2018-0013.

Kee, J.D., Rho, J.H., Kim, K.H., Lee, D.H, 2014: High Speed Driving Stability of Passenger Car Under Crosswind Effects. International Journal of Automotive Technology, Vol. 15, No. 5, pp. 741-747.

Kim, K., Kim, B., Go, Y., Park, J., Park, J., Suh, I., Yi, K., 2014. "An investigation on motor-driven power steering-based crosswind disturbance compensation for the reduction of driver steering effort", Vehicle System Dynamics: International Journal of Vehicle Mechanics and Mobility, 52:7, 922-947.

Mansor, S. and Passmore, M.A., 2008. Estimation of bluff body transient aerodynamics using an oscillating model rig. Journal of Wind Engineering and Industrial Aerodynamics, 96(6-7), pp. 1218-1231.

Nakasato, K., Tsubokura, M., Ikaeda, J., Onishi, K., Ota, S., Takase, H., Akasaka, K., Ihara, H., Oshima, M., Araki, T.: Coupled 6DoF Motion and Aerodynamic Crosswind Simulation Incorporating Driver Model. SAE Int. J. Passeng. Cars - Mech. Syst., 10(2), 662-670 (2017). 
Nakashima, T., Tsubokura, M., Vazquez, M., Owen, H., Doi, Y.: Coupled analysis of unsteady aerodynamics and vehicle motion of a road vehicle in windy conditions. Computers and Fluids, 80: 1-9 (2013). doi:10.1016/j.compfluid.2012.09.028

Schröck, D., Widdecke, N., Wiedemann, J., 2009. Aerodynamic Response of a Vehicle Model to Turbulent Wind. FKFS Conference, Stuttgart.

Shur, M.L., Spalart, P.R., Strelets, M.Kh., Travin, A.K.:A hybrid RANSLES approach with delayed-DES and wall-modelled LES capabilities. International Journal of Heat and Fluid Flow 29 (2008) 1638-1649. doi:10.1016/j.ijheatfluidflow.2008.07.001

Sims-Williams, D. (2011). "Cross winds and transients: reality, simulation and effects", SAE Paper No. 2011-01-0172.

Storms, B.L., Ross, J.C., Heineck,J.T., Walker S.M., Driver, D.M., Zilliac, G.G.: An experimental study of the ground transportation system (GTS) model in the NASA Ames 7-by 10-ft wind tunnel. NASA TM-2001-209621 (2001) 2001.

Theissen, P., 2012. "Unsteady Vehicle Aerodynamics in Gusty Crosswind", PhD thesis, Technische Universität München.

Tunay, T., O’Reilly, C., Drugge, L.: "The significance of roll on the dynamics of ground vehicles subjected to crosswind gusts by two-way coupled simulation of aero- and vehicle dynamics", 26th IAVSD Symposium on Dynamics of Vehicles on Roads and Tracks, 12-16 August 2019 Gothenburg, Sweden. 
Unaune, S.V., Sovani, S.D., Kim, S.E., 2005. Aerodynamics of a Generic Ground Transportation System: Detached Eddy Simulation. In: 2005 SAE World Congress Detroit, Michigan April 11-14, 2005.

Volpe, R., Ferrand, V., DaSilva, A., LeMoyne, L., 2014. Forces and flow structures evolution on a car body in a sudden crosswind. J. Wind Eng. Ind. Aerodyn. 128, 114-125. http://dx.doi.org/10.1016/j.jweia.2014.03.006.

Watkins, S., 1990. Wind-Tunnel Modelling of Vehicle Aerodynamics: With Emphasis on Turbulent Wind Effects on Commercial Vehicle Drag. PhD Thesis, Victorian University of Technology.

Watkins, S., Toksoy, S., Saunders, J., 1992. On the Generation of Tunnel Turbulence for Road Vehicles. 11th AFMC, Hobart, Australia, December, 1992.

Winkler, N., Drugge, L., Trigell, A.S., Efraimsson, G.: Coupling aerodynamics to vehicle dynamics in transient crosswinds including a driver model. Computers and Fluids, 138: 26-34 (2016). doi:10.1016/j.compfluid.2016.08.006

Wojciak J., 2012. "Quantitative analysis of vehicle aerodynamics during crosswind gusts", PhD thesis, Technische Universität München. 